\title{
Cognitive Influences on the Generation of Eye Movements
}

\author{
Rochelle Ackerley, ${ }^{1}$ Kelly Wild, ${ }^{1}$ and Alexis Makin ${ }^{2}$ \\ ${ }^{1}$ Faculty of Life Sciences, University of Manchester, Manchester M60 1QD, United Kingdom and ${ }^{2}$ School of Psychological Science, University of Manchester, \\ Manchester M13 9PL, United Kingdom \\ Review of de Hemptinne et al. (http://www.jneurosci.org/cgi/content/full/28/17/4298)
}

Smooth pursuit eye movements are influenced by both visual motion signals and extraretinal factors, such as expectation (Barnes and Asselman, 1991). In a recent microelectrode recording study, de Hemptinne et al. (2008) present evidence linking the supplementary eye fields (SEFs) of the rhesus monkey with the processing of expectations about the direction of target motion during smooth pursuit. The SEFs are located in the frontal cortex, dorsomedial to the frontal eye fields (FEFs) and were first documented by Schlag and Schlag-Rey (1985), who found that SEF neurons have directional firing preferences. SEF neurons exhibit increased activity before and during eye movements, particularly for saccades, but this has more recently been observed for smooth pursuit eye movements (Heinen, 1994). The work of de Hemptinne et al. (2008) expands our understanding of SEF function, by showing that some neurons in this region encode higher-level motion direction representations for the initiation and facilitation of smooth pursuit eye movements.

de Hemptinne et al. (2008) presented a single moving target to rhesus monkeys; the target moved either left or right. In

Received June 18, 2008; revised July 11, 2008; accepted July 18, 2008.

We thank Prof. Graham Barnes, Dr. Sue Collins, Dr. Emma Gowen, and

Dr. Ellen Poliakoff for constructive comments and discussions on this manuscript.

Correspondence should be addressed to Dr. Rochelle Ackerley, Faculty of Life Sciences, University of Manchester, Moffat Building, Sackville Street, P0 Box 88, Manchester M60 1QD, UK. E-mail: Rochelle. Ackerley@Manchester.ac.uk.

DOl:10.1523/JNEUROSCI.2802-08.2008

Copyright $\odot$ 2008 Society for Neuroscience $\quad 0270-6474 / 08 / 288863-02 \$ 15.00 / 0$ half of the trials, a colored directional cue was presented before motion onset, indicating the direction in which the upcoming target would move. In the other half, a neutral cue that did not predict upcoming target direction was presented. Cues were presented for $500 \mathrm{~ms}$ while the monkeys fixated. The delay between the cue and motion onset was 0,400 , or $600 \mathrm{~ms}$. Single-cell recordings were made in the SEF region, and eye movements were recorded throughout. When a directional cue was presented, monkeys produced anticipatory smooth pursuit eye movements in the cued direction. These responses were of higher velocity and were initiated earlier with respect to motion onset when delays were greater than zero.

Analysis was based on 174 of 413 SEF neurons in which activity was altered by the presence of a directional cue compared with a neutral cue. The responses of these neurons to the directional cue were explored during the interval between the cue and motion onset. Firing rate was altered in three ways: (1) activity increased when the cue indicated upcoming activity in either direction, and these neurons had no preferred direction before motion onset; (2) activity increased when the cue predicted upcoming motion in the "preferred" direction only, with no modulation in the opposite ("antipreferred") direction; or (3) activity increased when the cue indicated movement in the neuron's preferred direction, but decreased when the directional cue indicated upcoming motion in the antipreferred direction. The second type of modulation was the most prevalent. The majority of responding neurons $(76 \%)$ belonged to the second and third categories, indicating that SEF neurons have strong directional preferences. Firing rate increased further still after motion onset, when visual input was available; therefore, SEF neurons have a role in both the prediction of motion direction and the pursuit of a target.

When a neutral cue was presented without a delay, the cue elicited very few anticipatory eye movements. However, when a neutral cue was presented with a delay before motion onset, randomly orientated anticipatory smooth pursuit eye movements occurred during the delay interval. There was little change in neural activity in the SEF after a neutral cue with any of the delay durations used. In summary, firing in these SEF neurons increased after the presentation of a cue that predicted upcoming motion direction, but not when the cue predicted motion without specifying its direction. These cells therefore encoded expectations about the direction of upcoming motion.

An alternative explanation for some of the results reported by the authors is that neuronal activity reflected premotor activity signals involved in the execution of anticipatory smooth pursuit eye movements. These SEF neurons may have been simply relaying direction expectation signals computed by upstream neural populations. However, de Hemptinne et al. (2008) provide evidence against this. First, a significant correlation between the onset of cue-related activity and the onset of anticipatory eye movements was evi- 
dent in only $7 \%$ of the directionally sensitive neurons. The absence of tight temporal coupling between increased firing and the beginning of eye movement suggests that the activity is not simply a premotor signal. Second, randomly orientated smooth pursuit eye movements were produced in the neutral cue condition, but firing rate in the SEF cells did not rise above baseline in the majority of recorded neurons. This suggests that most were not involved in eye movement production itself and may thus be related to expectation. In future work, this hypothesis could be tested further by including a "no-go" task, identical to the cued direction condition, but with fixation maintained throughout. If SEF activity is truly related to cognitive expectation of upcoming motion direction rather than eye movement execution, then neural activity after the directional cue should be the same in the no-go task as in the "go" task.

How do the findings of de Hemptinne et al. (2008) fit with those of previous SEF investigations? Chen and Wise (1995) investigated the changes in SEF activity to learning a bidirectional visual target saccadic task. They found that SEF neurons had directional selectivity in horizontal and vertical planes, but these directional preferences were not constant or fixed, and responses changed during the process of task acquisition. Two main neuronal modulations to learning were found: learning-selective cases, in which neurons increased in activity during task acquisition, and learning-dependent cases, in which responses were highest once the task was familiar. de Hemptinne et al. (2008) did not investigate the learning process (their results reflected performance after training), or any responses in the vertical plane. This may explain why only $42 \%$ of all cells tested by de Hemptinne et al. (2008) responded to the directional cue, given that some SEF neurons may be recruited during task acquisition only, or may currently be involved in encoding non-horizontal directions. Never- theless, both studies add evidence to the role of the SEFs in the flexible sampling and storage of directional information to formulate expectations about learned oculomotor behavior.

Of course, the SEFs do not work independently of other brain regions. As de Hemptinne et al. (2008) mention, the SEFs are reciprocally connected to the FEFs. In a recent exploration of SEF-FEF interaction, Coe et al. (2002) used a freechoice saccade task and found that the SEF firing was altered before target presentation, before the FEF neurons. These authors concluded that anticipatory activity generated in the SEF reflects internal decision-making processes for upcoming oculomotor movement, which is then transmitted to the FEFs. Furthermore, it has been hypothesized that the SEFs are involved in craniocentric (eye in head) oculomotor control, whereas FEFs are involved in retinocentric (eye) oculomotor control only. This would mean that the SEFs also receive input from vestibular nuclei (Fukushima et al., 2004). Finally, other areas involved in motion perception and guiding smooth pursuit eye movements, such as medial temporal/medial superior temporal areas and various subregions of the intraparietal sulcus, are heavily connected to the SEFs (Nobre et al., 1999). How these anatomically distributed populations are functionally integrated, and what exact role they play during target tracking, is a question for future work.

From the current evidence, it seems likely that the SEFs are involved in encoding the direction of upcoming movement. The SEFs may therefore function as a type of working memory for oculomotor control; eye movements could be planned and facilitated by the SEFs by means of an internal representation of future movement acquired through experience. This idea could be further investigated by exploring the role of the SEFs in anticipating upcoming target velocity. After all, anticipatory smooth pursuit eye movements are influenced not only by expectation of upcoming movement in a particular direction, but also by the expectation of upcoming movement at a particular velocity (Barnes and Asselman, 1991). Indeed, Fukushima et al. (2004) suggest that the SEFs encode some types of velocity and vestibular information, but the SEF activity appears to be largely task dependent. In addition, the relationship between SEF activity and head movement control could be explored because, along with eye movements, anticipatory head movements are often involved in target tracking, necessitating suppression of the vestibulo-ocular reflex by pursuit-related and other, nonvisual, mechanisms (Fukushima et al., 2004).

\section{References}

Barnes GR, Asselman PT (1991) The mechanism of prediction in human smooth pursuit eye movements. J Physiol 439:439-461.

Chen LL, Wise SP (1995) Neuronal activity in the supplementary eye field during acquisition of conditional oculomotor associations. J Neurophysiol 73:1101-1121.

Coe B, Tomihara K, Matsuzawa M, Hikosaka O (2002) Visual and anticipatory bias in three cortical eye fields of the monkey during an adaptive decision-making task. J Neurosci 22:5081-5090.

Fukushima J, Akao T, Takeichi N, Kurkin S, Kaneko CR, Fukushima K (2004) Pursuitrelated neurons in the supplementary eye fields: discharge during pursuit and passive whole body rotation. J Neurophysiol 91:2809-2825.

Heinen SJ (1994) Evidence of a timing mechanism for predictive smooth pursuit in frontal cortex. In: Contemporary ocular motor and vestibular research: a tribute to David. A. Robinson (Fuchs AF, Brandt T, Buttner U, Zee D, eds), pp 408-410. New York: Thieme.

Nobre AC, Coull JT, Frith CD, Mesulam MM (1999) Orbitofrontal cortex is activated during breaches of expectation in tasks of visual attention. Nat Neurosci 2:11-12.

Schlag J, Schlag-Rey M (1985) Unit activity related to spontaneous saccades in frontal dorsomedial cortex of monkey. Exp Brain Res 58: 208-211. 